\title{
Oncogenic roles of TOPK and MELK, and effective growth suppression by small molecular inhibitors in kidney cancer cells
}

\author{
Taigo Kato ${ }^{1}$, Hiroyuki Inoue ${ }^{1}$, Seiya Imoto², Yoshinori Tamada², Takashi Miyamoto ${ }^{3}$, \\ Yo Matsuo ${ }^{3}$, Yusuke Nakamura ${ }^{1,4}$ and Jae-Hyun Park ${ }^{1}$ \\ ${ }^{1}$ Department of Medicine, The University of Chicago, Chicago, IL, USA \\ 2 Human Genome Center, Institute of Medical Science, The University of Tokyo, Tokyo, Japan \\ ${ }^{3}$ OncoTherapy Science Inc., Kawasaki, Japan \\ ${ }^{4}$ Department of Surgery, The University of Chicago, Chicago, IL, USA \\ Correspondence to: Yusuke Nakamura, email: ynakamura@bsd.uchicago.edu \\ Keywords: kidney cancer, TOPK, MELK, molecular target, kinase inhibitor \\ Received: November 25, 2015 Accepted: February 09, $2016 \quad$ Published: February 26, 2016
}

\section{ABSTRACT}

T-lymphokine-activated killer cell-originated protein kinase (TOPK) and maternal embryonic leucine zipper kinase (MELK) have been reported to play critical roles in cancer cell proliferation and maintenance of stemness. In this study, we investigated possible roles of TOPK and MELK in kidney cancer cells and found their growth promotive effect as well as some feedback mechanism between these two molecules. Interestingly, the blockade of either of these two kinases effectively caused downregulation of forkhead box protein M1 (FOXM1) activity which is known as an oncogenic transcriptional factor in various types of cancer cells. Small molecular compound inhibitors against TOPK (OTS514) and MELK (OTS167) effectively suppressed the kidney cancer cell growth, and the combination of these two compounds additively worked and showed the very strong growth suppressive effect on kidney cancer cells. Collectively, our results suggest that both TOPK and MELK are promising molecular targets for kidney cancer treatment and that dual blockade of OTS514 and OTS167 may bring additive anti-tumor effects with low risk of side effects.

\section{INTRODUCTION}

Kidney cancer is the seventh most common cancer and the tenth most common cause of cancer death in men, and it is also the tenth most common cause of cancer in women [1]. In 2015, the number of new kidney and renal pelvis cancer cases was estimated to be 61,560 that led to more than 14,080 deaths in United States [1]. The 5-year disease-specific survival has improved from about $50 \%$ in $1975-1977$ to $65 \%$ in 2000-2005, although that of patients at an advanced stage still remains poorly with around $10 \%$ rate [2-4]. $30 \%$ of patients who underwent surgery of localized kidney cancer develop distant metastasis and have limited therapeutic options, such as tyrosine kinase inhibitor (TKI) and mammalian target of rapamycin (mTOR) inhibitors [5]. In addition, the clinical effects of these drugs are very limited and patients often discontinue administration of these drugs due to severe side effects including hand-foot syndrome, liver dysfunction and interstitial pneumonia [6-10]. Therefore, development of more effective therapy for kidney cancer is eagerly expected.

TOPK (T-lymphokine-activated killer celloriginated protein kinase, also known as PBK or PDZbinding kinase) is a Ser/Thr protein kinase that is highly expressed in various types of human cancer [11-14]. TOPK is known to be activated during the cell mitosis process and play a critical role in cytokinesis. We previously reported that knockdown of TOPK caused dysfunction of cytokinesis and subsequently apoptosis of cancer cells [13].

MELK (maternal embryonic leucine zipper kinase, also known as MPK38 or murine protein serine/threonine kinase 38) is a cell-cycle dependent protein kinase that belongs to the AMP-activated Ser/Thr protein kinase family [15, 16]. Elevated expression of the MELK gene is correlated with poorly differentiated histological types of brain tumor and prostate cancer $[17,18]$, and with poor 
prognosis of breast cancer patients [19].

The two molecules, TOPK and MELK, have shown similar expression patterns; they are up-regulated in various types of cancer including cancer stem cellenriched tumors and more importantly their expressions are hardly detectable in normal organs except in the testis $[11,20]$. Moreover, MELK expression levels were strongly correlated with those of forkhead box protein M1 (FOXM1) known as an important transcriptional factor and a master regulator of mitosis in cancer stem cells $[21,22]$. These results suggest a possible close link among TOPK, MELK, and FOXM1 in a growth regulation pathway in cancer cells, which may provide a new strategy for successful treatment of cancer patients. Hence, we have developed TOPK inhibitors (OTS514 and OTS964) and a MELK inhibitor (OTS167) that showed therapeutic potentials in pre-clinical models of human cancer [23, 24].

In the present study, we demonstrate that TOPK regulates FOXM1 like as MELK does and that knockdown of either TOPK or MELK effectively suppresses the growth signaling pathway composed of these three oncoproteins. We also demonstrated that the combination of OTS514 and OTS167 can effectively reduce the expression levels of TOPK, MELK and FOXM1, and decreased viability of kidney cancer cells. These findings suggest that dual blockade using a combination of a TOPK inhibitor (OTS514) and a MELK inhibitor (OTS167) at the lower dose may be a promising molecular-targeted therapy for kidney cancer patients with avoidance or reduction of their toxicity.

\section{RESULTS}

\section{TOPK and MELK expression in kidney cancer cell lines}

We examined expression levels of TOPK and MELK genes in kidney cancers through publically-available gene expression datasets. The Oncomine database revealed that both TOPK and MELK genes are significantly upregulated in kidney cancers (Supplementary Figure S1). Interestingly, the Cancer Genome Atlas (TCGA) data showed that expression levels of TOPK and MELK are strongly correlated in various cancer types as shown in Supplementary Figure S2, suggesting that TOPK and $M E L K$ may be regulated by a common transcription pathway or may be in some positive feedback loop [2527]. Based on these findings, we investigated expression levels of TOPK and MELK in 16 kidney cancer cell lines by western blot analysis (Figure 1A). Although some cell lines showed the discordance in TOPK and MELK protein levels, most of the cell lines examined revealed the concordant expression levels, further suggesting some interaction between TOPK and MELK.

\section{Knockdown effects of endogenous TOPK and MELK}

To investigate the biological function of TOPK and MELK in kidney cancer cells, we used siRNA (small interfering RNA) to knockdown TOPK and MELK expression using three kidney cancer cell lines, VMRCRCW, Caki-1, and Caki-2 in which TOPK and MELK were highly co-expressed (Figure 1A). Each of siRNA successfully knocked down the transcript levels of the target genes (Figure 1B) and also significantly reduced the amount of its target protein (Figure 1C). However, unexpectedly, knockdown of TOPK led to reduction of MELK protein level and vice versa knockdown of MELK reduced TOPK protein level (Figure 1C). The semi-quantitative RT-PCR revealed that the expression of $M E L K$ was also downregulated by TOPK knockdown and vice versa knockdown of MELK downregulated TOPK transcription level (Figure 1B), suggesting that TOPK and MELK are likely to be influenced each other.

\section{TOPK and MELK knockdown downregulates FOXM1 activity}

The transcriptional interaction between $T O P K$ and $M E L K$ allowed us to examine any possible transcriptional factor that can influence on expression of these two genes. In the TCGA database, we found that TOPK and $M E L K$ expression levels were strongly correlated with that of FOXM1 (Pearson's rank correlation is 0.73 and 0.82 , respectively, Supplementary Figure S3, 4) [26, 27]. Moreover, we previously reported that the MELK inhibitor reduced expression of FOXM1 at protein level [22]. FOXM1 is a key transcriptional factor involved in the proliferation of cancer cells including leukemia cells that were very sensitive to both TOPK and MELK inhibitors [22, 28]. It is also notable that TOPK, MELK, and FOXM1 were suggested as cancer stem cell markers and listed in top 30 of the "consensus stemness ranking (CSR) signature" genes [29]. Based on these findings, we examined whether knockdown of TOPK or MELK could influence on the expression of FOXM1. As expected, FOXM1 protein level was decreased in kidney cancer cells transfected with siMELK (Figure 2A), probably due to the decrease of FOXM1 transcription (Figure 2B). Intriguingly, siTOPK also resulted in the downregulation of FOXM1 not only in protein level but also in transcriptional level (Figure 2A and 2B).

\section{Influence of FOXM1 on TOPK and MELK expression}

Because FOXM1 was reported to bind promoter regions of TOPK and MELK genes in chromatin 
A

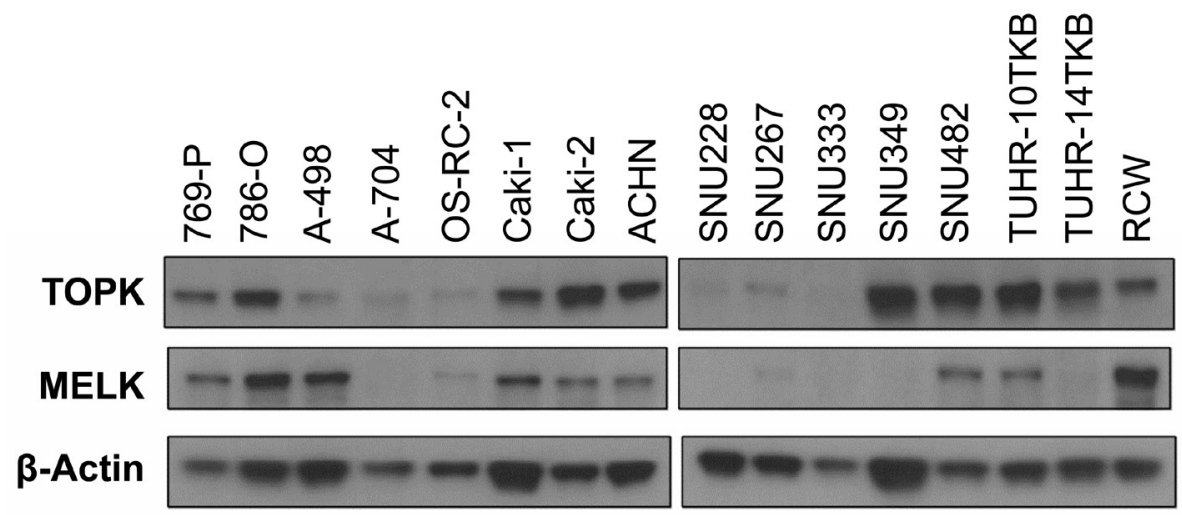

B

VMRC-RCW

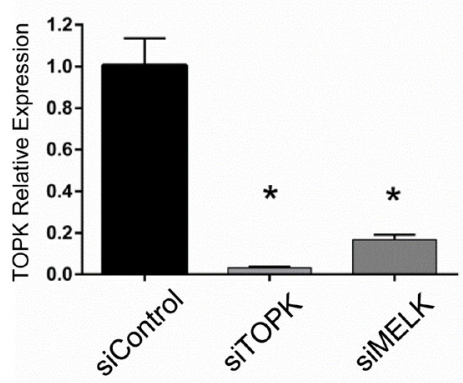

VMRC-RCW

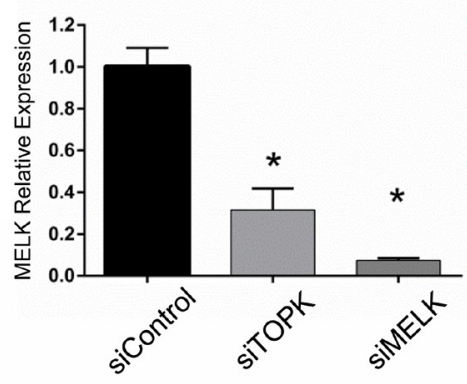

C

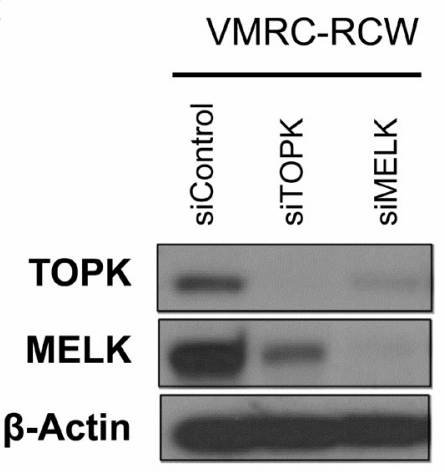

Caki-1

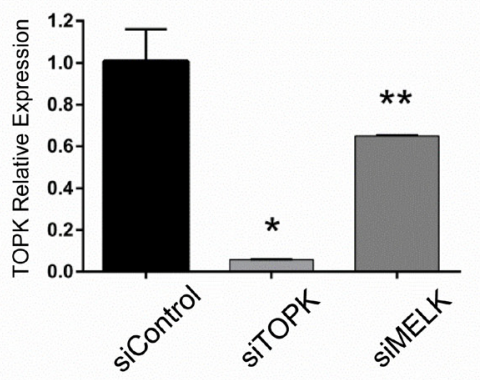

Caki-1

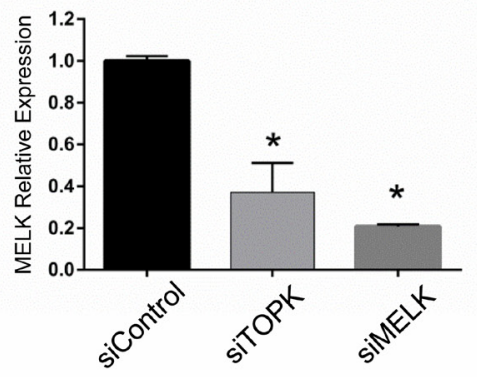

Caki-1

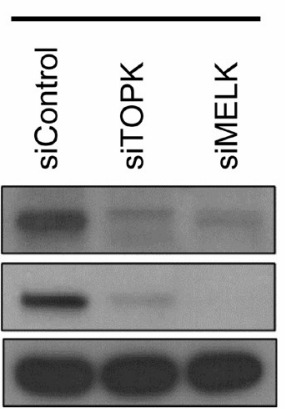

Caki-2

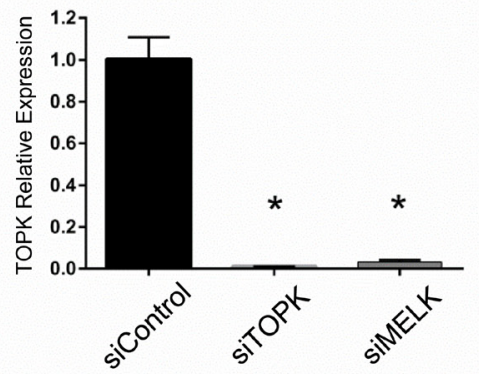

Caki-2

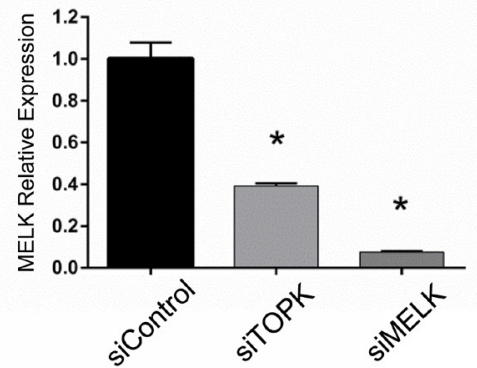

Caki-2

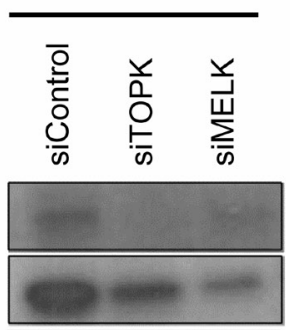

$\beta$-Actin

Figure 1: Expression and knockdown effects of TOPK and MELK in kidney cancer cell lines. A. Expression of endogenous TOPK and MELK protein in 16 kidney cancer cell lines examined by Western blot analysis. B. The transcriptional level of MELK was downregulated by TOPK knockdown with siTOPK. MELK knockdown also led to downregulation of TOPK in the transcriptional level. C. Silencing of TOPK expression with siTOPK also reduced the MELK expression in kidney cancer cell lines. TOPK expression was also suppressed by MELK knockdown with siMELK. ${ }^{*} p<0.01,{ }^{* *} p<0.05$ compared with the corresponding value of the siControl group. 
immunoprecipitation (ChIP) assay [30], we investigated transcriptional regulation on the TOPK and MELK genes by FOXM1. We first transfected kidney cancer cells with SiFOXM1 and found that both TOPK and MELK mRNA levels were decreased in three kidney cancer cell lines compared with the cells treated with control siRNA (Figure 3A). We then exogenously introduced FOXM1 expression vector into two kidney cancer cell lines and found the significant elevation of TOPK and MELK mRNA expression in the cells transfected with FOXM1 expression vectors, while no change was observed in those transfected with the mock control vector (Figure 3B). Taken together, these results suggest that FOXM1 may function as a transcriptional factor that induces expression of TOPK and MELK genes in kidney cancer cells. Since either TOPK or MELK suppression with siRNA downregulated FOXM1, the feedback system among these three genes seems to be very complicated.

\section{Growth suppressive effect of OTS514 and OTS167 in kidney cancer cells}

Since both $T O P K$ and MELK were overexpressed in kidney cancer $[26,27]$, we examined the half-maximum inhibitory concentration $\left(\mathrm{IC}_{50}\right)$ value to measure the growth inhibitory effect of OTS514 and OTS167 on five kidney cancer cell lines, VMRC-RCW, Caki-1, Caki-2, 769-P and 786-O, in which TOPK and MELK were highly co-expressed (Figure 4A-4E). Our assay revealed low $\mathrm{IC}_{50}$ values as 19.9 to $44.1 \mathrm{nM}$ for TOPK inhibitor OTS514 and 12.4 to $38.7 \mathrm{nM}$ for MELK inhibitor OTS167.

\section{Downregulation of TOPK, MELK and FOXM1 by OTS514 and OTS167}

Our previous study in leukemic cells showed that downregulation of MELK resulted in the suppression of
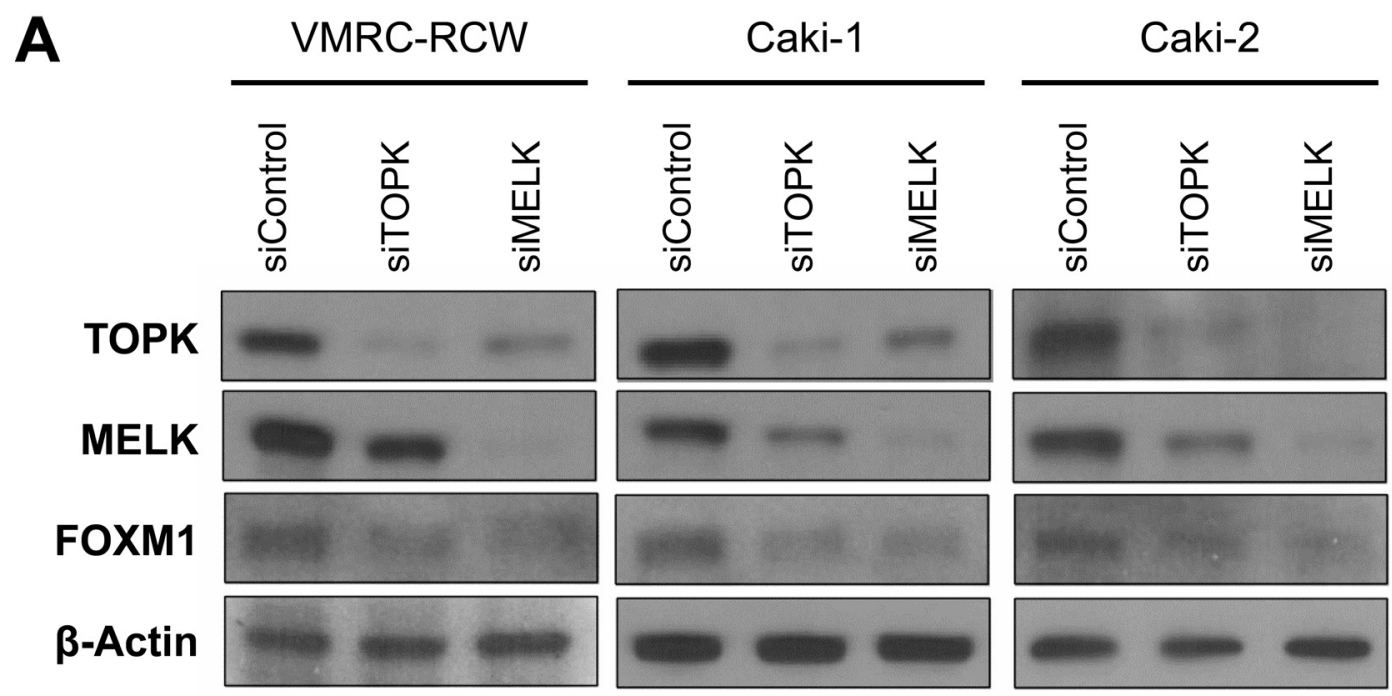

B

VMRC-RCW

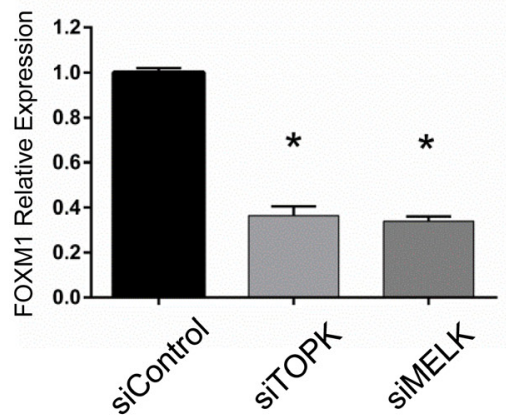

Caki-1

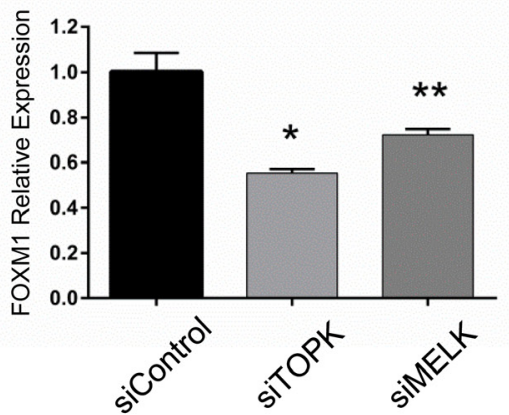

Caki-2

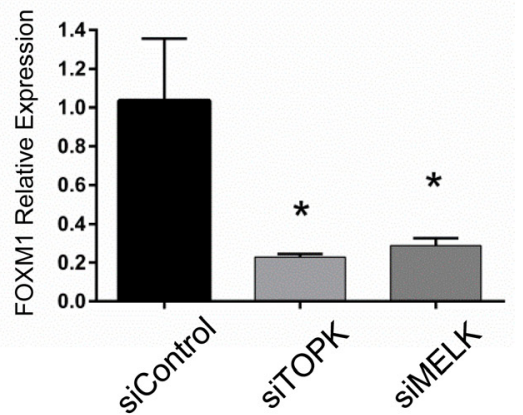

Figure 2: Both TOPK and MELK regulate expression of FOXM1. A. Silencing of TOPK expression downregulated FOXM1 protein level as similar as MELK affected on it in kidney cancer cell lines. B. Both siTOPK and siMELK downregulated FOXM1 expression in the transcriptional level. ${ }^{*} p<0.01,{ }^{*} p<<0.05$ compared with the corresponding value of the siControl group. 
A
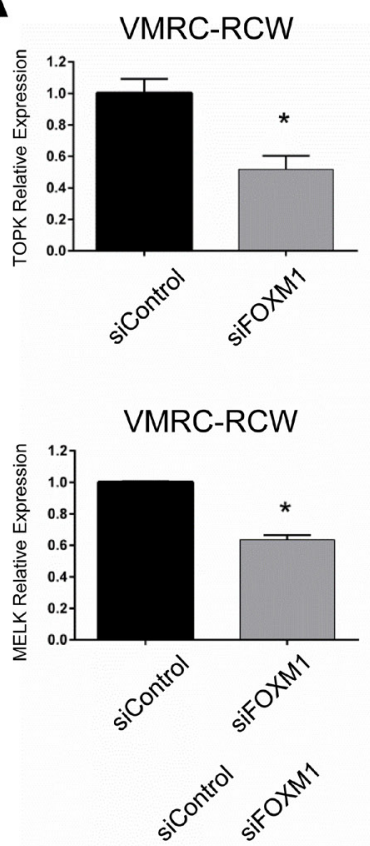

FOXM1

$\beta$-Actin

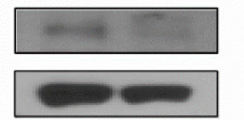

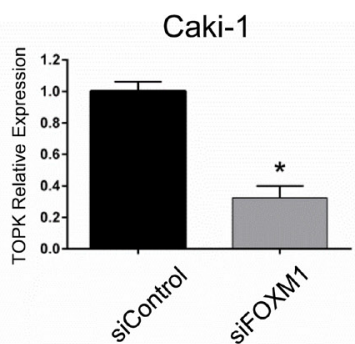

B

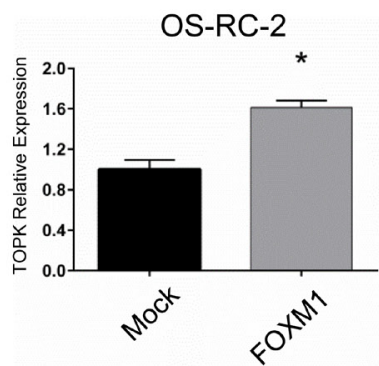

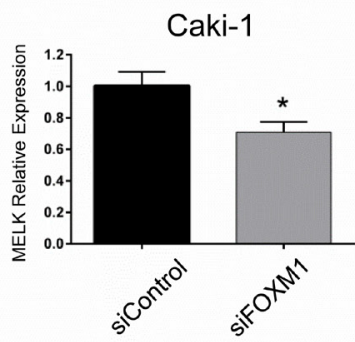
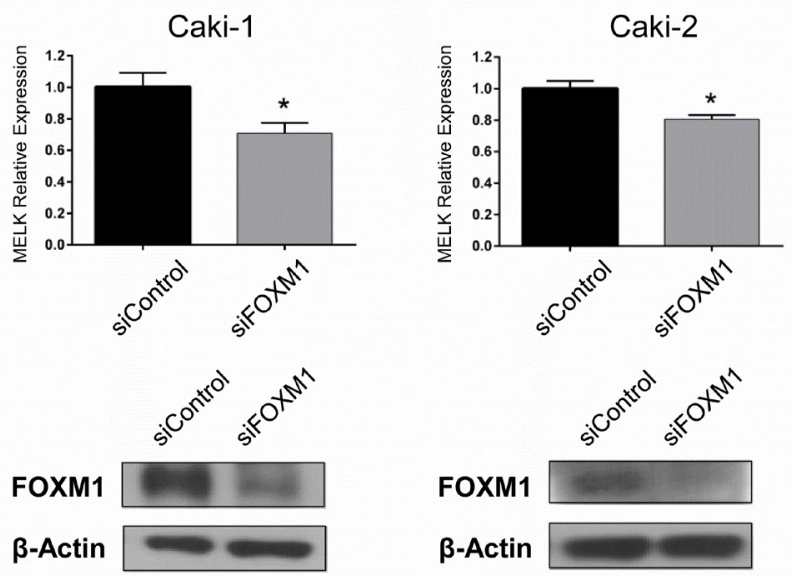

Caki-2

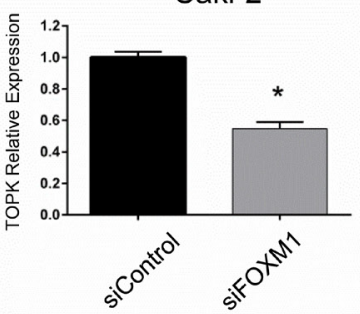

Caki-2

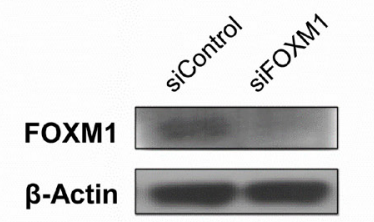

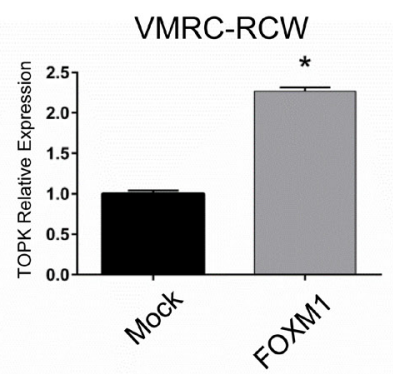
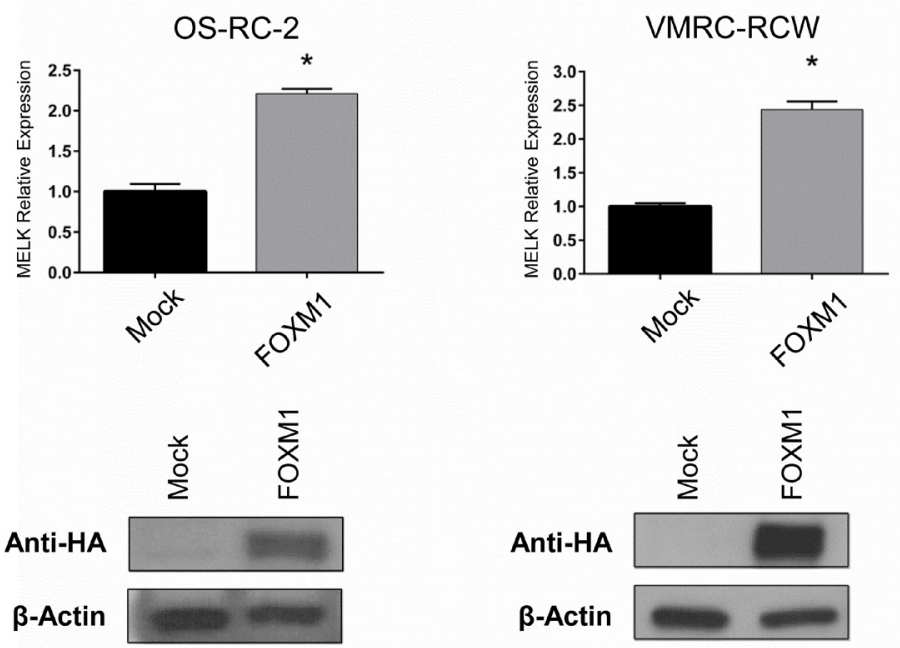

Figure 3: FOXM1 also regulates TOPK and MELK expression. Expression level of TOPK and $M E L K$ were examined by RTPCR after depletion or overexpression of FOXM1 in kidney cancer cells. A. FOXM1 knockdown led to the downregulation of TOPK and $M E L K$ expression in transcriptional levels. Western blots showed depletion of FOXM1 in kidney cancer cells 48 hrs after transfection with siFOXM1. B. The exogenous expression of FOXM1 was confirmed by western blot using anti-HA antibody, 48 hrs after transfection. The asterisk indicates $p<0.01$ compared with the corresponding value of the siControl group. 
FOXM1 [22]. Similarly, our results in kidney cancer cells treated with the $\mathrm{IC}_{50}$ concentration of MELK inhibitor (OTS167) showed decrease of MELK and TOPK proteins as well as FOXM1 protein (Figure 5). This downregulation effect of three proteins by OTS167 was in concordant with that of siMELK treatment (Figure. 2A). Interestingly, treatment with TOPK inhibitor (OTS514) at the $\mathrm{IC}_{50}$ condition also remarkably reduced the protein level of all three proteins (Figure 5), similar to the results obtained by siTOPK treatment (Figure 2A). Collectively, our assays implied that both inhibitors are very effective to suppress kidney cancer cell growth through reduction of TOPK, MELK, and FOXM1 proteins that may cooperatively constitute a critical signal pathway in kidney cancer cells.

\section{Dual TOPK and MELK inhibition additively reduced cell viability and increased apoptosis}

Although a detailed molecular mechanism in regulation of TOPK, MELK, and FOXM1 needs to be clarified, our findings from siRNA experiments and assays using small molecule inhibitors strongly indicated some kind of feedback loop machinery among the three molecules. Taking into consideration of clinical application, we evaluated a combinatory inhibitory effect against TOPK and MELK. We transfected the low amount of siRNA (50 pmol each) that is equivalent to one-fourth of siRNA that we used knockdown assays shown in Figure 1. Although this amount of siRNA was capable to reduce $T O P K$ and $M E L K$ expression modestly, combination of siTOPK and siMELK at this concentration resulted in much stronger effect on reduction of TOPK and MELK expression as well as FOXM1 expression (Figure 6A).
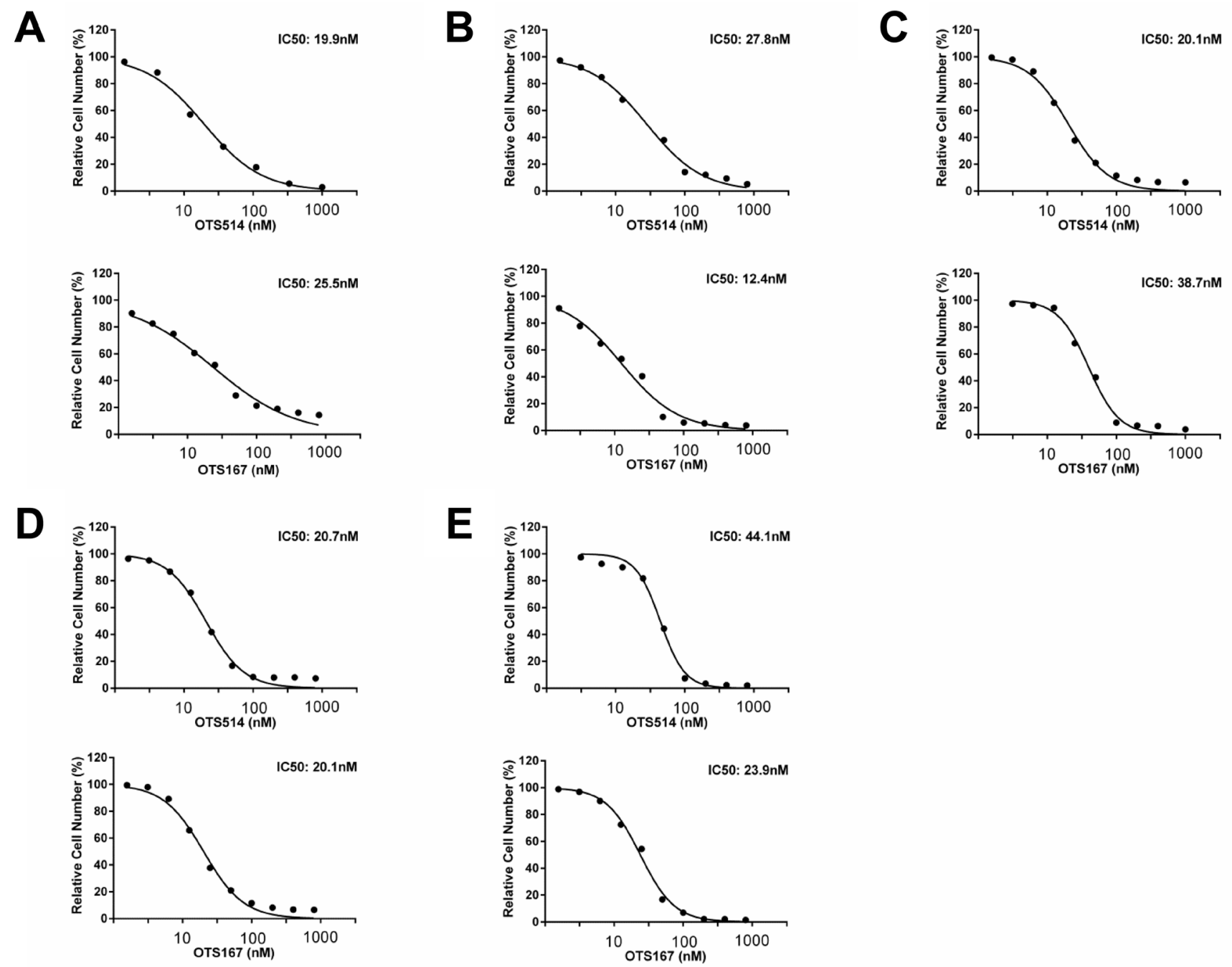

Figure 4: In vitro anti-proliferative effects of OTS514 and OTS167 in kidney cancer cell lines. In vitro anti-proliferative effects of OTS514 and OTS167 in kidney cancer cell lines. Each graph shows growth inhibition curves of OTS514 and OTS167 for kidney cancer cell lines, VMRC-RCW A., Caki-1 B., Caki-2 C., 769-P D., and 786-O E., in which both TOPK and MELK proteins are highly expressed. 
Concordantly, combination of siTOPK and siMELK effectively achieved decrease of cell viability of kidney cancer cells (Figure 6B). Subsequently, three kidney cancer cell lines were treated with $\mathrm{IC}_{50}$ values of OTS514 or/and OTS167. As expected, MTT assay revealed that the combination treatment of OTS514 and OTS167 significantly decreased cell viability for all of the kidney cancer cells examined, compared with the treatment with a single compound (Figure 7A). In addition, we assessed apoptosis of cancer cells (Caki-2) by flow cytometry and observed significantly increased apoptosis of $14.7 \pm 2.6$ $\%(p=0.001$, for OTS514) or $10.6 \pm 2.6 \%(p=0.001$, for OTS167) at an early time-point of drug treatment (Annexin-positive but PI-negative field) when cells were treated with the two drugs (Figure 7B). Consistent with these findings, the cleaved caspase 3 was significantly increased in the cells with the combination of OTS514 and OTS167 ( $p=0.03$ and $p=0.02$, respectively, Figure 7C). Effective induction of apoptosis by combination of two compounds was also observed in kidney cancer VMRCRCW cells (Supplementary Figure S5).

\section{DISCUSSION}

Despite advances in the novel treatment modalities, 5-year cancer specific survival rate of kidney cancer patients who are at the advanced stage still remains around $10 \%[1,2,4]$. This low rate may be partially explained by the poor response of kidney cancer to chemotherapy and radiation therapy. Moreover, patients who once relapsed or are at stage IV have limited therapeutic options such as kinase inhibitors and mTOR inhibitors, which often cause severe adverse effects [5,6]. Given the limitation of these therapeutic choices, novel effective anti-cancer drugs should be developed urgently.

According to the Oncomine database, TOPK and $M E L K$ are upregulated in kidney cancer and considered as promising molecular targets because of their cancerrestricted expression patterns [26, 27]. Previous studies have shown that MELK inhibition effectively reduced expression of an oncogenic transcriptional factor, FOXM1 [22]. In the present study, we further demonstrated that TOPK knockdown also caused suppression of FOXM1 expression as similar to MELK knockdown. We also revealed that the growth of kidney cancer was suppressed by either TOPK or MELK inhibition.

The transcriptional interaction among TOPK, MELK and FOXM1 seems to be very complicated. We have demonstrated that treatment of cancer cells with either siTOPK or siMELK downregulated FOXM1 both in protein level and in transcriptional level. These results implied the presence of some feedback mechanism that regulates expression of the FOXM1 gene. To elucidate this complex feedback system, we conducted large-scale gene network analysis by the SiGN-BN algorithm using a supercomputer system. However, we could not find any possible candidate factors involved in the MELK-TOPKFOXM1 pathway in any deposited cancer database. This may be partially explained by (1) lack of relevant kidney cancer dataset in the database, (2) indirect regulation of these three genes in the feedback loop including multiple

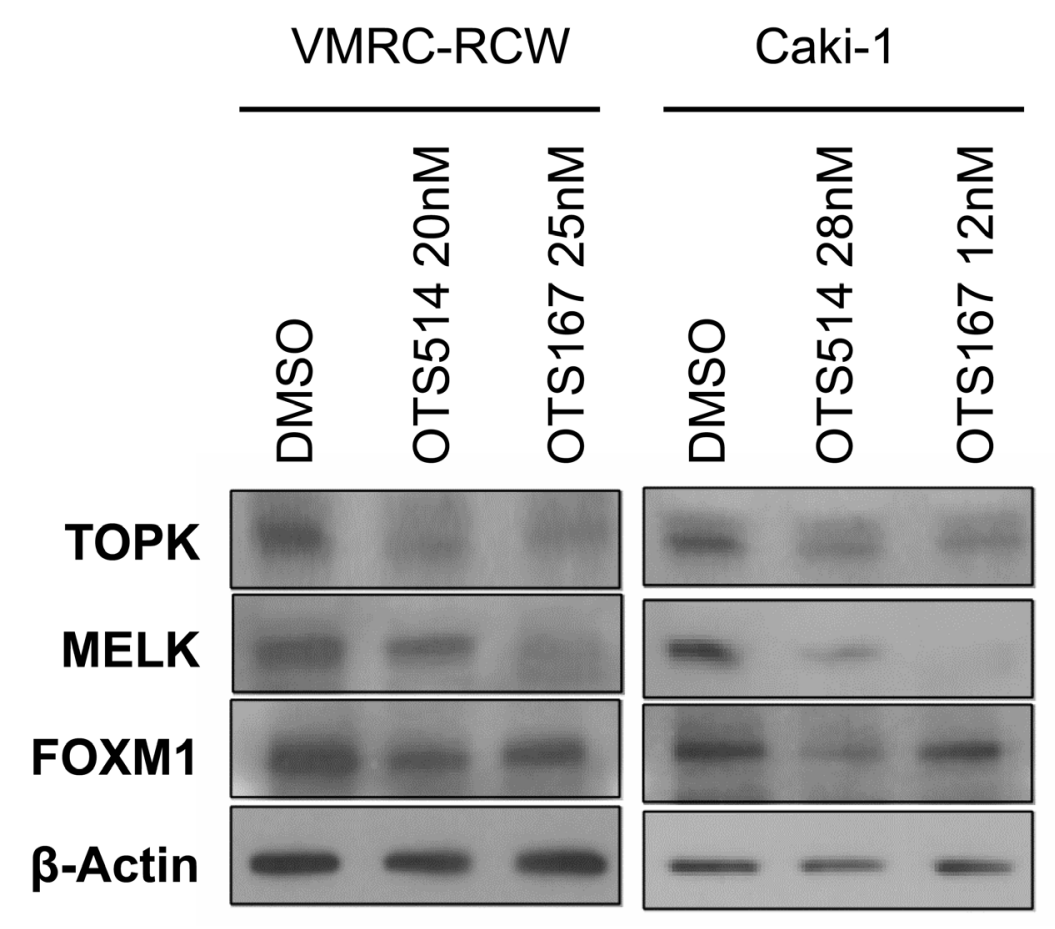

Figure 5: Downregulation of FOXM1 by OTS514 and OTS167 treatment. Treatment with OTS514 or OTS167 at the concentration of their $\mathrm{IC}_{50}$ values reduced FOXM1 protein level in VMRC-RCW and Caki-1 cells, as examined by western blot analysis. 
mediator genes, or (3) both. Although further molecular mechanism should be elucidated, our findings suggested the existence of a complex feedback loop among
TOPK, MELK and FOXM1. Because knockdown of TOPK and MELK revealed much stronger effect on the growth suppression of kidney cancer cells than that of
A

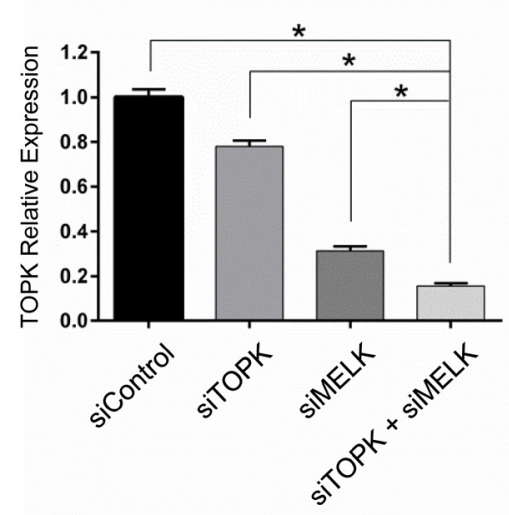

Caki-2

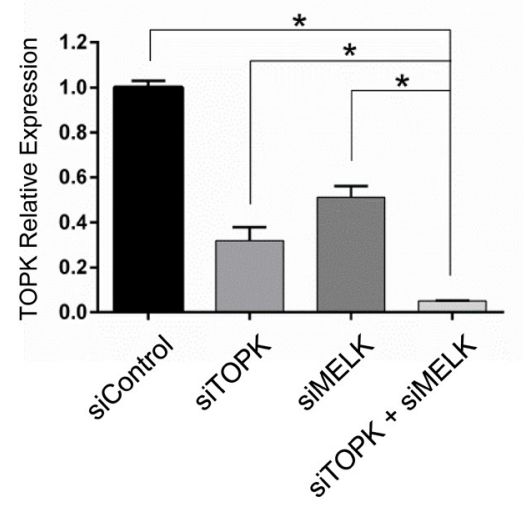

VMRC-RCW

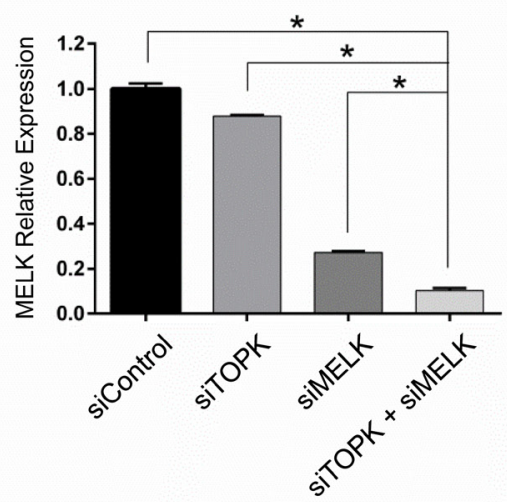

Caki-2

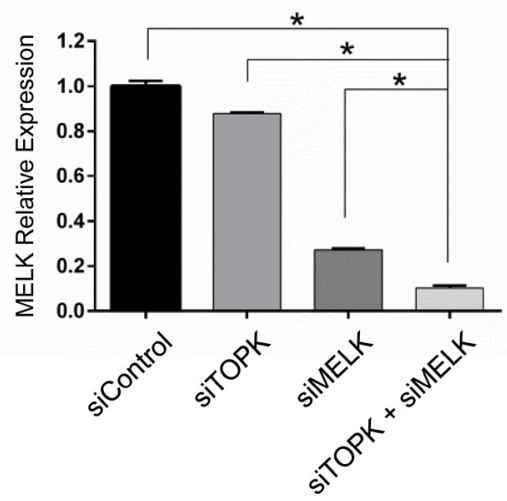

VMRC-RCW

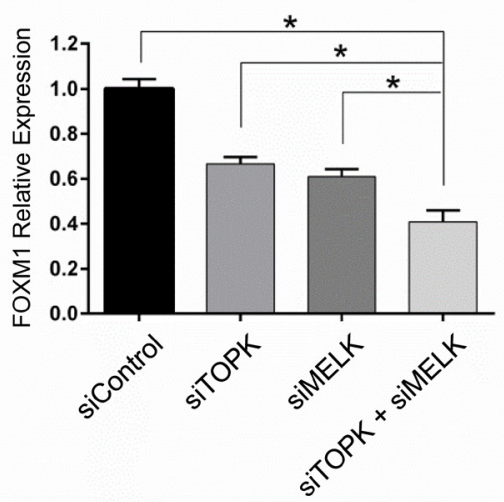

Caki-2

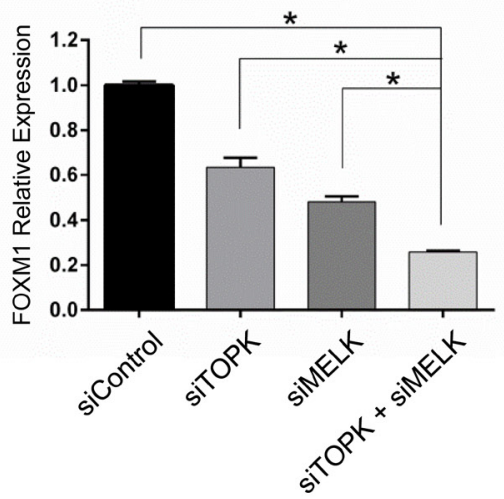

B

VMRC-RCW

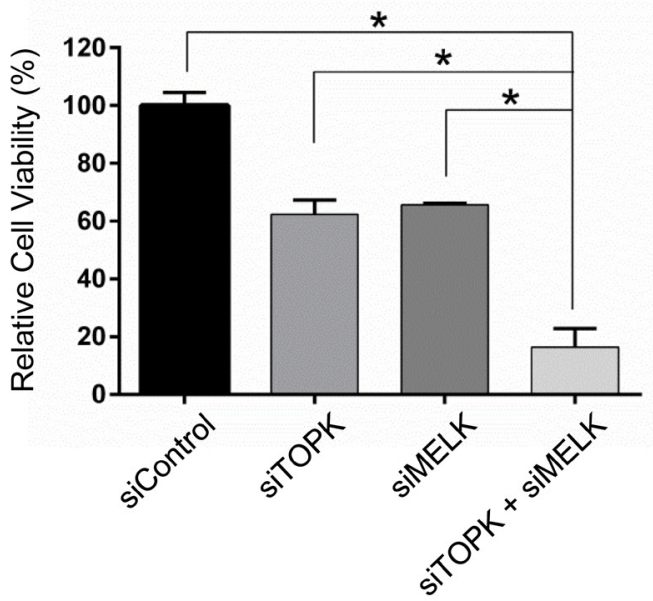

Caki-2

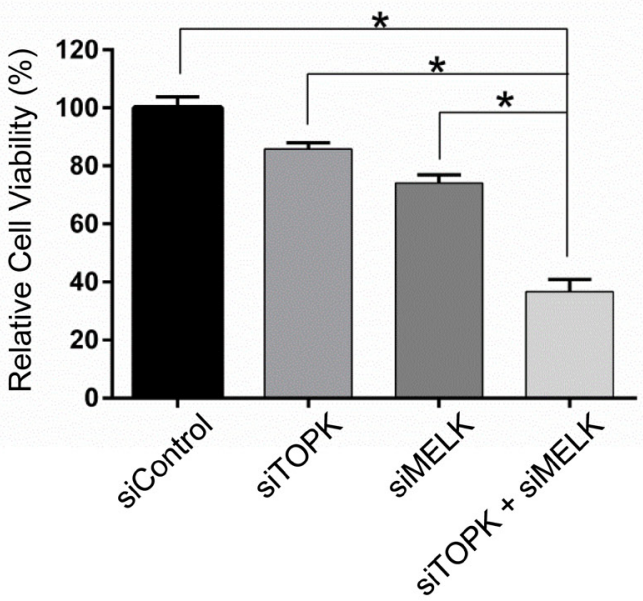

Figure 6: Additive effects with dual TOPK and MELK inhibition with low-dose siRNAs in kidney cancer cells. A. Dual knockdown with low-dose siTOPK (50 pmol) + siMELK (50pmol) additively suppressed TOPK, MELK and FOXM1 expression in transcriptional level compared with either knockdown of TOPK or MELK alone. B. Dual knockdown of TOPK and MELK with low-dose siRNAs showed drastic decrease in the cell viability compared with either knockdown of TOPK or MELK alone. The asterisk indicates $p$ $<0.01$ compared with the corresponding value of the siControl group. 
A

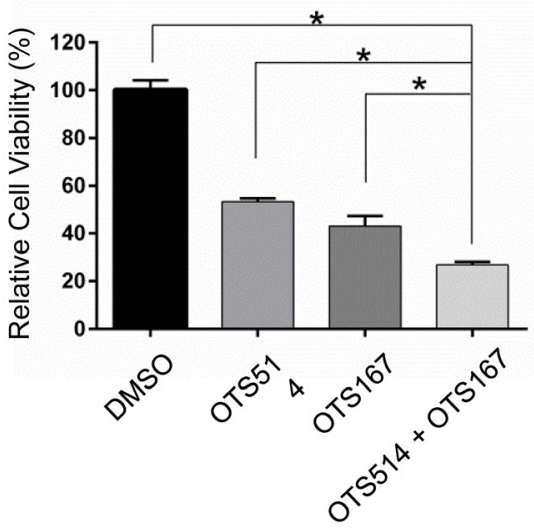

B
Caki-1

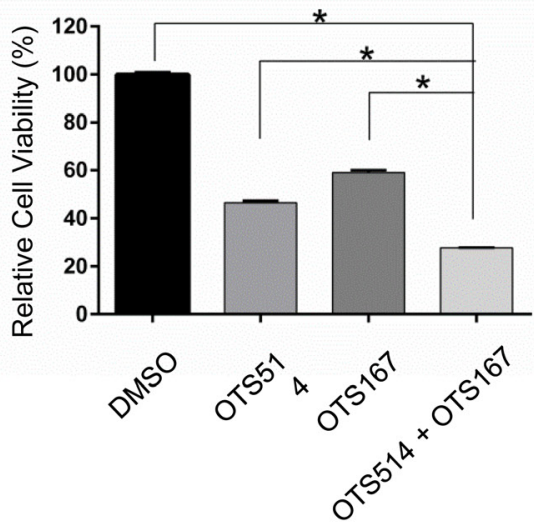

Caki-2

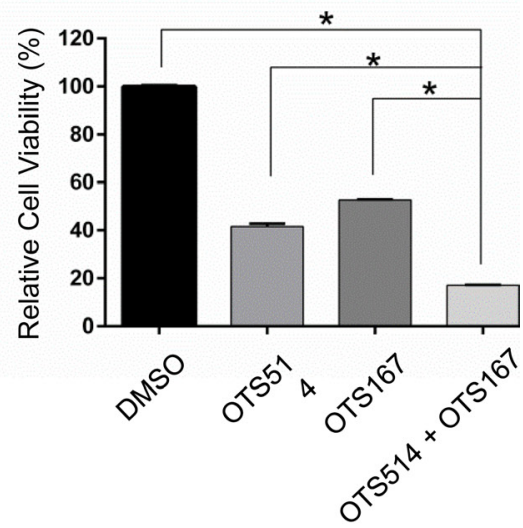

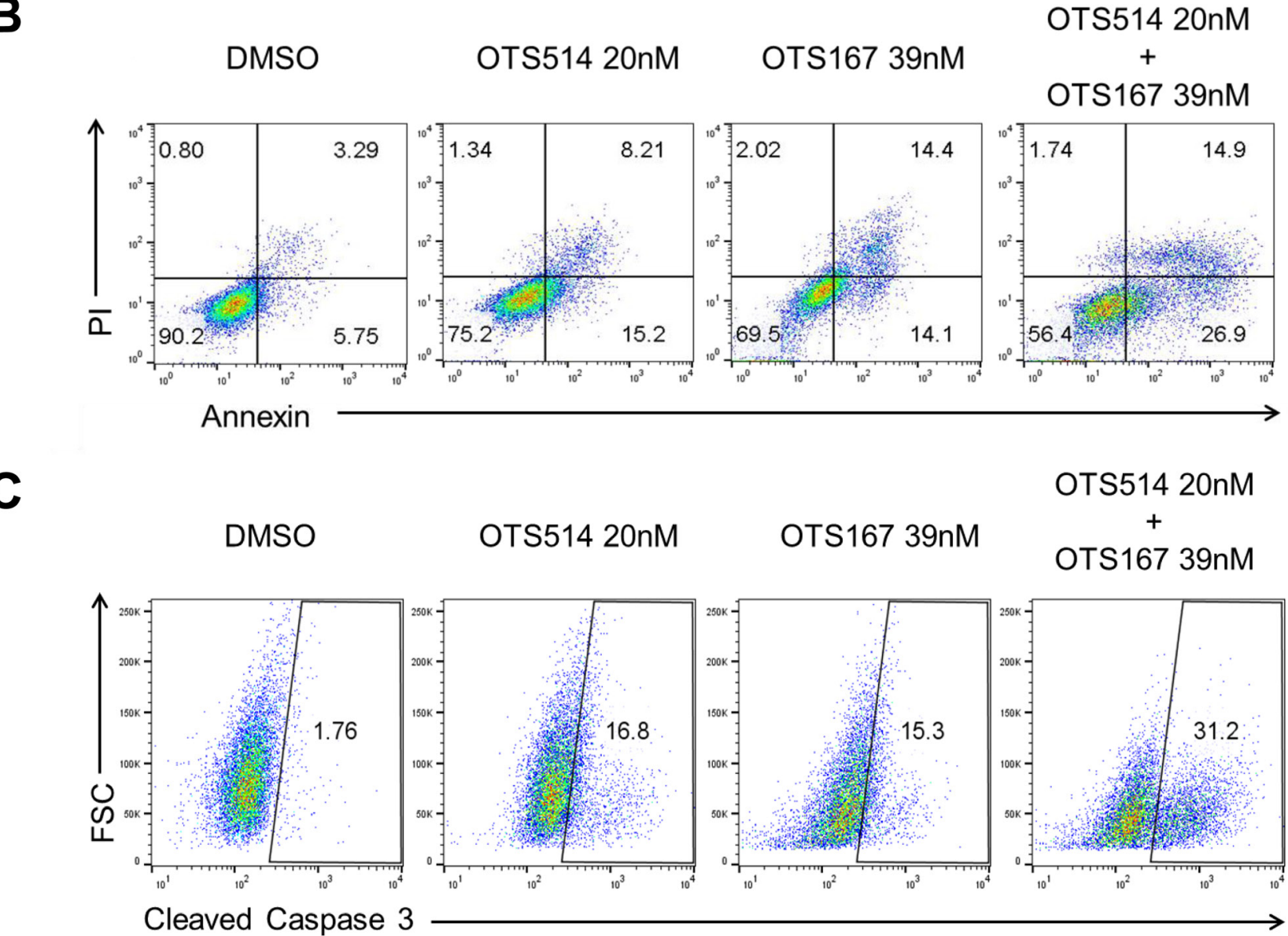

Figure 7: The effects in the decrease of kidney cancer cell viability by combination of OTS514 and OTS167. A. The combination of OTS514 and OTS167 at the concentration of $\mathrm{IC}_{50}$ values significantly revealed growth-suppressive effects on kidney cancer cells compared with the treatment of a single compound. The asterisk indicates $p<0.01$ compared with the treatment of a single compound. B. Early stage of apoptosis assessed by Annexin and PI staining of kidney cancer cells. The combination of OTS514 and OTS167 at the concentration of $\mathrm{IC}_{50}$ values significantly increased $14.7 \pm 2.6 \%$ (compared with OTS514 alone) or $10.6 \pm 2.6 \%$ (compared with OTS167 alone) of apoptotic cells $(p=0.001$ and $p=0.001$, respectively). C. Apoptosis was examined by the levels of cleaved caspase 3 in kidney cancer cells. Dual inhibition with OTS514 and OTS167 significantly increased $10.8 \pm 3.1 \%$ (compared with OTS514 alone) or $12.5 \pm$ $2.9 \%$ (compared with OTS167 alone) of late stage population of apoptosis compared with either inhibition alone ( $p=0.03$ and $p=0.02$, respectively). Flow cytometry figures are representative of three independent experiments. 
FOXM1 (data not shown), it is almost certain that TOPK and MELK are more attractive molecular targets than FOXM1 to inhibit signaling pathways essential for cancer proliferation, although it remains unclear which will serve as an upstream of others and play more fundamental roles in kidney cancer.

Pre-clinical studies showed that treatment with TOPK inhibitor (OTS964, a derivative of OTS514) could induce complete regression of tumors but led to adverse reactions in hematopoietic cells [23]. On the other hand, MELK inhibitor (OTS167) did not cause any adverse reactions at the effective dose although it did not result in complete regression of tumors in mice model [24]. In fact, we demonstrated that dual blockade with low doses of TOPK inhibitor (OTS514) and MELK inhibitor (OTS167) achieved the additive cancer cell killing effects rather compared with the single treatment. Considering advantages and disadvantages of these compounds, it will be essential to figure out the most effective combination dose to achieve complete tumor elimination with minimum risk of adverse reaction.

In summary, we suggest that both TOPK and MELK are attractive molecular targets for kidney cancer treatment and they constitute a feedback loop with an oncogenic transcriptional factor FOXM1. Given that the beneficial effects of dual administration of low dose OTS514 and OTS167, this combination strategy will provide more potential cancer therapeutics that could be applied to a various types of human malignancy.

\section{MATERIALS AND METHODS}

\section{Cell lines, plasmids, oligo siRNAs and transfection}

769-P, 786-O, A-498, A-704, Caki-1, Caki-2 and ACHN cells were purchased from the American Type Culture Collection (ATCC; Manassas, VA). OS-RC-2, TUHR-10TKB and TUHR-14TKB cells were provided by RIKEN BioResource Center (Tsukuba, Japan). VMRCRCW was provided by the Cell Resource Center for Biomedical Research, Institute of Development, Aging and Cancer, Tohoku University. SNU228, SNU267, SNU333, SNU349 and SNU482 were provided by Dr. JaeGahb Park (Korean Cell Line Bank, Seoul, Korea). All cells were cultured under appropriate media recommended by suppliers with $10 \%$ FBS and $1 \%$ antibiotic-antimycotic solution (Sigma-Aldrich, St. Louis, MO). All cells were maintained at $37^{\circ} \mathrm{C}$ in humidified air with $5 \% \mathrm{CO}_{2}$. For knockdown experiments, cells were transfected with 200 pmol or 50 pmol (low dose experiment) of oligo siRNA using Lipofectamine RNAiMAX (Invitrogen, Carlsbad, CA) according to manufacturer's instructions. The target sequences of oligo siRNAs were as follows: 5'-GUGUGGCUUGCGUAAAUAA-3' for TOPK;
5'-GACAUCCUAUCUAGCUGCA-3' for MELK; and 5'-GGACCACUUUCCCUACUU-3' for FOXM1. To construct vectors designed to express FOXM1 (NM_021953.3), the entire coding sequences were amplified by RT-PCR and cloned into the pCAGGSnHc expression vector. Plasmids were transfected using FugeneHD (Roche, Basel, Switzerland) according to the supplier's recommendations.

\section{Western blot analysis and antibodies}

Western blot analysis was performed by normalization to $\beta$-actin or the baseline expression level. Cells were lysed with IP lysis buffer (Thermo Scientific, Waltham, MA) containing protease inhibitor cocktail III (Millipore, Billerica, MA). The proteins were separated by electrophoresis using 4-20\% or 7.5\% SDS-PAGE gel, and transferred onto nitrocellulose membrane. The membranes were incubated with the first antibody, respectively: antiTOPK antibody (BD Biosciences, San Jose, CA), antiFOXM1 antibody (Santa Cruz Biotechnology, Santa Cruz, CA), anti-HA (Roche), or anti- $\beta$-actin (Sigma-Aldrich). Finally, the membrane was incubated with horseradish peroxidase-conjugated secondary antibody and protein bands were visualized by enhanced chemiluminescence detection reagents (GE Healthcare, Pittsburgh, PA). We generated mouse anti-MELK monoclonal antibodies using partial recombinant MELK protein (264-601 amino acids of MELK) as an immunogen by the methods as described previously [31].

\section{Cell viability assay}

For methyl thiazolyl tetrazolium (MTT) assay, cancer cells were seeded into 24-well flat-bottom plates (BD Falcon) at $5 \times 10^{4}$ cells per well, and mixed with oligo siRNA or TOPK and/or MELK inhibitors. Compounds of OTS514 and OTS167 were kindly provided by OncoTherapy Science Inc. (Kawasaki, Japan). Cells were cultured at $37^{\circ} \mathrm{C}$ under $5 \% \mathrm{CO}_{2}$ for $72 \mathrm{~h}$. The Cell counting kit-8 (Dojindo Molecular Technologies, Inc., Kumamoto, Japan) was used for MTT reaction and examined the cell viability. After reaction for 1 to $3 \mathrm{hr}, 100$ $\mu \mathrm{L}$ of supernatant was transferred into a 96 -well plate and read in a microplate reader at $450 \mathrm{~nm}$. For the viability and apoptosis analyses, cells were collected, spun down then washed with PBS and resuspended in $50 \mu \mathrm{L}$ of binding buffer containing $2 \mu \mathrm{L}$ of Annexin V (eBioscience, San Diego, CA). After 20 min incubation, cells were stained with $100 \mu \mathrm{L}$ of binding buffer containing $1 \mu \mathrm{L}$ propidium iodide (PI) (eBioscience). For a cleaved caspase 3 assay, cells were collected, spun down, then washed with PBS, resuspended in $500 \mu \mathrm{L}$ Cytofix/Cytoperm solution (eBioscience) and incubated cells on ice for $20 \mathrm{~min}$. Subsequently, cells were spun down, washed with Perm/ 
Wash buffer (eBioscience) and resuspended with $100 \mathrm{uL}$ of buffer containing $20 \mu \mathrm{L}$ of cleaved caspase 3 antibody (eBioscience). Fluorescence was quantified by flow cytometry (FACS LSRII; Becton Dickinson, San Jose, CA). Flow Jo software (Treestar, Ashland, OR) was used to identify Annexin and PI positive cell subpopulations and cleaved caspase 3 - positive cell subpopulations.

\section{Real-time RT-PCR}

Total RNA was extracted from cell monolayers using RNeasy Mini Kit (Qiagen, Valencia, CA) according to the manufacturer's directions. Total RNA $(1-2 \mu \mathrm{g})$ was reversely transcribed using SuperScript III FirstStrand Synthesis System (Invitrogen) following the manufacturer's instructions. Aliquots of the reverse transcription product were quantified by real-time RTPCR. The RT-PCR was performed using primers listed below using the ViiA 7 system (Life Technologies, Grand Island, NY). The expression levels were normalized with that of $G A P D H$. The PCR primer sequences were 5'-AGACCCTAAAGATCGTCCTTCTG-3' and 5'-GTGTTTTAAGTCAGCATGAGCAG-3' for TOPK; 5'-GCTGCAAGGTATAATTGATGGA-3' and 5'-CAGTAACATAATGACAGATGGGC-3' for MELK; 5'-CAACCGCTACTTGACATTGGA-3' and 5'-TCACCGGGAACTGGATAGG-3' for FOXM1 and 5'-CGACCACTTTGTCAAGCTCA-3' and 5'-GGTTGAGCACAGGGTACTTTATT-3' for GAPDH.

\section{Gene network analysis}

For the gene network analysis, gene expression datasets deposited in the NCBI GEO were analyzed by SiGN-BN software with supercomputer "K computer" in Advanced Institute for Computational Science Riken, Japan [32, 33]. The resulting networks were compiled as a gene network databases, The Cancer Network Galaxy (TCNG, http://tcng.hgc.jp/). We searched for sumnetworks containing three genes, TOPK, MELK and $F O X M 1$ and their first and second neighbors.

\section{Statistical analysis}

Data were expressed as mean \pm one standard deviation. Differences between two groups were examined for significance using student's $t$ test. Differences were considered significant at $p<0.05$. Experiments were performed in triplicate.

\section{ACKNOWLEDGMENTS}

We thank Drs. Suyoun Chung, Yosuke Harada, Kenji Tamura and Houda Alachkar for helpful discussions about oncogenic function of MELK and characteristics of OTS compounds.

\section{GRANT SUPPORT}

This work was supported in part by a research grant from OncoTherapy Science Inc.

\section{CONFLICTS OF INTEREST}

Y.N. is a stockholder and an adviser of OncoTherapy Science Inc. J.P. is a scientific adviser of OncoTherapy Science Inc. T.M. and Y.M. are employees of OncoTherapy Science Inc.

\section{Authors' contributions}

Y.N. planned and supervised the entire project; J.P. provided the study design and the working hypothesis and completed the article; T.K. designed the study, conducted experiments, performed data analysis, and drafted the article; S.I. and Y.T. conducted gene network data analysis and H.I. conducted cell apoptosis experiments and provided scientific advice. T.M. and Y.M. provided OTS compounds and supplementary materials for cellbased assays.

\section{REFERENCES}

1. American Cancer Society. Cancer Facts and Figures 2015. Atlanta: American Cancer Society 2015.

2. Gandaglia G, Ravi P, Abdollah F, Abd-El-Barr AE, Becker A, Popa I, Briganti A, Karakiewicz PI, Trinh QD, Jewett MA and Sun M. Contemporary incidence and mortality rates of kidney cancer in the United States. Can Urol Assoc J. 2014; 8: 247-252.

3. Chaffer CL and Weinberg RA. A perspective on cancer cell metastasis. Science. 2011; 331: 1559-1564.

4. Novara G, Ficarra V, Antonelli A, Artibani W, Bertini $\mathrm{R}$, Carini $\mathrm{M}$, Cosciani Cunico $\mathrm{S}$, Imbimbo $\mathrm{C}$, Longo $\mathrm{N}$, Martignoni G, Martorana G, Minervini A, Mirone $\mathrm{V}$, et al. Validation of the 2009 TNM version in a large multi-institutional cohort of patients treated for renal cell carcinoma: are further improvements needed? Eur Urol. 2010; 58: 588-595.

5. Pichler M, Hutterer GC, Chromecki TF, Jesche J, Kampel-Kettner K, Rehak P, Pummer K and Zigeuner R. External validation of the Leibovich prognosis score for nonmetastatic clear cell renal cell carcinoma at a single European center applying routine pathology. J Urol. 2011; 186: 1773-1777.

6. Banumathy $G$ and Cairns P. Signaling pathways in renal cell carcinoma. Cancer Biol Ther. 2010; 10: 658-664.

7. Lai JS, Beaumont JL, Diaz J, Khan S and Cella D. 
Validation of a short questionnaire to measure symptoms and functional limitations associated with hand-foot syndrome and mucositis in patients with metastatic renal cell carcinoma. Cancer. 2016; 122: 287-95. doi: 10.1002/ cncr.29655.

8. Suttle AB, Ball HA, Molimard M, Hutson TE, Carpenter C, Rajagopalan D, Lin Y, Swann S, Amado R and Pandite L. Relationships between pazopanib exposure and clinical safety and efficacy in patients with advanced renal cell carcinoma. Br J Cancer. 2014; 111: 1909-1916.

9. Ivanyi P, Fuehner T, Adam M, Eichelberg C, Herrmann E, Merseburger AS, Ganser A and Grunwald V. Interstitial lung disease during targeted therapy in metastatic renal cell carcinoma: a case series from three centres. Med Oncol. 2014; $31: 147$.

10. Willemsen AE, Grutters JC, Gerritsen WR, van Erp NP, van Herpen CM and Tol J. mTOR inhibitor-induced interstitial lung disease in cancer patients: Comprehensive review and a practical management algorithm. Int J Cancer. 2016; 138:2312-21. doi: 10.1002/ijc.29887.

11. Park JH, Lin ML, Nishidate T, Nakamura $Y$ and Katagiri T. PDZ-binding kinase/T-LAK cell-originated protein kinase, a putative cancer/testis antigen with an oncogenic activity in breast cancer. Cancer Res. 2006; 66: 9186-9195.

12. Shih MC, Chen JY, Wu YC, Jan YH, Yang BM, Lu PJ, Cheng HC, Huang MS, Yang CJ, Hsiao M and Lai JM. TOPK/PBK promotes cell migration via modulation of the PI3K/PTEN/AKT pathway and is associated with poor prognosis in lung cancer. Oncogene. 2012; 31: 2389-2400.

13. Park JH, Nishidate $T$, Nakamura $Y$ and Katagiri $T$. Critical roles of T-LAK cell-originated protein kinase in cytokinesis. Cancer Sci. 2010; 101: 403-411.

14. Komatsu M, Yoshimaru T, Matsuo T, Kiyotani K, Miyoshi Y, Tanahashi T, Rokutan K, Yamaguchi R, Saito A, Imoto S, Miyano S, Nakamura Y, Sasa M, et al. Molecular features of triple negative breast cancer cells by genomewide gene expression profiling analysis. Int J Oncol. 2013; 42: 478-506.

15. Heyer BS, Warsowe J, Solter D, Knowles BB and Ackerman SL. New member of the Snf1/AMPK kinase family, Melk, is expressed in the mouse egg and preimplantation embryo. Mol Reprod Dev. 1997; 47: 148-156.

16. Badouel C, Chartrain I, Blot J and Tassan JP. Maternal embryonic leucine zipper kinase is stabilized in mitosis by phosphorylation and is partially degraded upon mitotic exit. Exp Cell Res. 2010; 316: 2166-2173.

17. Marie SK, Okamoto OK, Uno M, Hasegawa AP, ObaShinjo SM, Cohen T, Camargo AA, Kosoy A, Carlotti CG, Jr., Toledo S, Moreira-Filho CA, Zago MA, Simpson AJ, et al. Maternal embryonic leucine zipper kinase transcript abundance correlates with malignancy grade in human astrocytomas. Int J Cancer. 2008; 122: 807-815.

18. Kuner R, Falth M, Pressinotti NC, Brase JC, Puig SB, Metzger J, Gade S, Schafer G, Bartsch G, Steiner E,
Klocker H and Sultmann H. The maternal embryonic leucine zipper kinase (MELK) is upregulated in high-grade prostate cancer. J Mol Med (Berl). 2013; 91: 237-248.

19. Pickard MR, Green AR, Ellis IO, Caldas C, Hedge VL, Mourtada-Maarabouni M and Williams GT. Dysregulated expression of Fau and MELK is associated with poor prognosis in breast cancer. Breast Cancer Res. 2009; 11: R60.

20. Lin ML, Park JH, Nishidate T, Nakamura Y and Katagiri T. Involvement of maternal embryonic leucine zipper kinase (MELK) in mammary carcinogenesis through interaction with Bcl-G, a pro-apoptotic member of the Bcl-2 family. Breast Cancer Res. 2007; 9: R17.

21. Joshi K, Banasavadi-Siddegowda Y, Mo X, Kim SH, Mao P, Kig C, Nardini D, Sobol RW, Chow LM, Kornblum HI, Waclaw R, Beullens M and Nakano I. MELK-dependent FOXM1 phosphorylation is essential for proliferation of glioma stem cells. Stem Cells. 2013; 31: 1051-1063.

22. Alachkar $\mathrm{H}$, Mutonga MB, Metzeler $\mathrm{KH}$, Fulton $\mathrm{N}$, Malnassy G, Herold T, Spiekermann K, Bohlander SK, Hiddemann W, Matsuo Y, Stock W and Nakamura Y. Preclinical efficacy of maternal embryonic leucine-zipper kinase (MELK) inhibition in acute myeloid leukemia. Oncotarget. 2014; 5: 12371-12382. doi: 10.18632/ oncotarget.2642.

23. Matsuo Y, Park JH, Miyamoto T, Yamamoto S, Hisada $\mathrm{S}$, Alachkar H and Nakamura Y. TOPK inhibitor induces complete tumor regression in xenograft models of human cancer through inhibition of cytokinesis. Sci Transl Med. 2014; 6: 259ra145.

24. Chung S, Suzuki H, Miyamoto T, Takamatsu N, Tatsuguchi A, Ueda K, Kijima K, Nakamura Y and Matsuo Y. Development of an orally-administrative MELK-targeting inhibitor that suppresses the growth of various types of human cancer. Oncotarget. 2012; 3: 1629-1640. doi: 10.18632/oncotarget.790.

25. Kandoth C, McLellan MD, Vandin F, Ye K, Niu B, Lu C, Xie M, Zhang Q, McMichael JF, Wyczalkowski MA, Leiserson MD, Miller CA, Welch JS, et al. Mutational landscape and significance across 12 major cancer types. Nature. 2013; 502: 333-339.

26. Gumz ML, Zou H, Kreinest PA, Childs AC, Belmonte LS, LeGrand SN, Wu KJ, Luxon BA, Sinha M, Parker AS, Sun LZ, Ahlquist DA, Wood CG, et al. Secreted frizzled-related protein 1 loss contributes to tumor phenotype of clear cell renal cell carcinoma. Clin Cancer Res. 2007; 13: 47404749 .

27. Beroukhim R, Brunet JP, Di Napoli A, Mertz KD, Seeley A, Pires MM, Linhart D, Worrell RA, Moch H, Rubin MA, Sellers WR, Meyerson M, Linehan WM, et al. Patterns of gene expression and copy-number alterations in von-hippel lindau disease-associated and sporadic clear cell carcinoma of the kidney. Cancer Res. 2009; 69: 4674-4681.

28. Alachkar H, Mutonga M, Malnassy G, Park JH, Fulton N, Woods A, Meng L, Kline J, Raca G, Odenike O, Takamatsu 
N, Miyamoto T, Matsuo Y, et al. T-LAK cell-originated protein kinase presents a novel therapeutic target in FLT3ITD mutated acute myeloid leukemia. Oncotarget. 2015; 6: 33410-33425. doi: 10.18632/oncotarget.5418.

29. Shats I, Gatza ML, Chang JT, Mori S, Wang J, Rich J and Nevins JR. Using a stem cell-based signature to guide therapeutic selection in cancer. Cancer Res. 2011; 71: 17721780.

30. Sanders DA, Ross-Innes CS, Beraldi D, Carroll JS and Balasubramanian S. Genome-wide mapping of FOXM1 binding reveals co-binding with estrogen receptor alpha in breast cancer cells. Genome Biol. 2013; 14: R6.

31. Fukukawa C, Hanaoka H, Nagayama S, Tsunoda $T$, Toguchida J, Endo K, Nakamura $\mathrm{Y}$ and Katagiri $\mathrm{T}$. Radioimmunotherapy of human synovial sarcoma using a monoclonal antibody against FZD10. Cancer Sci. 2008; 99: 432-440.
32. Tamada $\mathrm{Y}$, Imoto S, Araki H, Nagasaki M, Print C, Charnock-Jones DS and Miyano S. Estimating genomewide gene networks using nonparametric Bayesian network models on massively parallel computers. IEEE/ACM Trans Comput Biol Bioinform. 2011; 8: 683-697.

33. Tamada Y, Shimamura T, Yamaguchi R, Imoto S, Nagasaki $\mathrm{M}$ and Miyano S. Sign: large-scale gene network estimation environment for high performance computing. Genome Inform. 2011; 25: 40-52. 